to appear ApJ, 597, Nov 10, 2003

\title{
Modeling Helical Structures in Relativistic Jets
}

\author{
Philip E. Hardee \\ Department of Physics 8 Astronomy, The University of Alabama, Tuscaloosa, AL 35487 \\ hardee@athena.astr.ua.edu
}

\begin{abstract}
Many jets exhibit twisted helical structures. Where superluminal motions are detected, jet orientation and pattern/flow speed are considerably constrained. In this case modeling efforts can place strong limits on conditions in the jet and in the external environment. This can be done by modeling the spatial development of helical structures which are sensitively dependent on these conditions. Along an expanding jet this sensitivity manifests itself in predictable changes in pattern speed and observed wavelength. In general, twists of low frequency relative to the local resonant frequency are advected along the expanding jet into a region in which the twist frequency is high relative to the local resonant frequency. The wave speed can be very different in these two frequency regimes. Potential effects include helical twists with a nearly constant apparent wavelength, an apparent wavelength scaling approximately with the jet radius for up to two orders of magnitude of jet expansion, or multiple twist wavelengths with vastly different intrinsic scale and vastly different wave speeds that give rise to similar observed twist wavelengths but with very different observed motion. In this paper I illustrate the basic intrinsic and observed behavior of these structures and show how to place constraints on jet conditions in superluminal jets using the apparent structures and motions in the inner $3 \mathrm{C} 120$ jet.
\end{abstract}

Subject headings: galaxies: jets — hydrodynamics — relativity — galaxies: active galaxies: individual (3C 120)

\section{Introduction}

The resolved highly collimated relativistic AGN jets can exhibit twisted time-dependent structures. For example, the 3C 345 jet shows non-radial curved motions and accelerations that have been interpreted as motions along a helix lying on the surface of a cone (Zensus, Cohen \& Unwin 1995; Steffen et al. 1995), the jet in M 87 shows helically twisted structures on a conical jet at parsec and hundreds of parsec scales (Reid et al. 1989; Owen et al. 1989) and apparent motions from subluminal to superluminal (Biretta, Sparks, \& Macchetto 1999), and the 3C 120 jet exhibits subluminal and superluminal apparent motions and intensity structure that has been interpreted as a helical twist on a conically expanding jet (Gómez et al. 1998; Walker et al. 2001). With the spatial and time resolution of present observations, we can begin to discern details such as the trajectory of individual components, the velocities of components and larger scale structures, and whether there are similarities between component speeds, trajectories, and larger scale structures.

Helical structure in relativistic jets can arise as a result of ordered variation in the flow direction at the central engine, e.g., precession, and/or as a result of random perturbations to the jet flow, e.g, jet cloud interaction (Gómez et al. 2000). Initial perturbations propagate as waves associated with pinch, helical, elliptical etc. normal mode distortions of the jet. The helically twisted structure of a jet is dependent on the initial excited wave frequency or frequencies and initial amplitudes, 
and on the subsequent propagation and growth or damping of these wave frequencies along the jet. The resulting helical structure can sensitively depend on jet speed via the Lorentz factor, on the sound speeds in the jet and surrounding material, and on the rate of jet expansion.

Only in the past few years have time dependent relativistic hydrodynamical codes become readily available (Duncan \& Hughes 1994; Martí, Müller, \& Ibáñez 1994; Koide, Nishikawa, \& Mutel 1996; Falle \& Komissarov 1996; Aloy et al. 1999a). More recently this has allowed fully $3 \mathrm{D}$ relativistic jet simulations to be performed with resolution sufficient to compare structure with theoretical predictions and/or with features observed in AGN jets (Aloy et al. 1999b, 2000; Hardee et al. 2001; Hughes et al. 2002; Hardee \& Hughes 2003; Aloy et al. 2003). In general, computational constraints make it very difficult to simulate and model the superluminal jets that must lie near to the line of sight. Simulations need to be conducted on lengthy computational grids and store many time steps in order to account for projection and light travel time effects, e.g., Gómez et al. (1997) and Aloy et al. (2003). This difficulty can be overcome using theoretical models based on the linearized fluid equations.

That the the normal wave modes predicted by the theory operate on AGN jets has been demonstrated by Lobanov \& Zensus (2001) who successfully fitted the twisted emission threads observed in the 3C 273 jet by a combination of helical and elliptical surface and internal normal modes. Other similar fitting has been performed by Lobanov, Hardee, \& Eilek (2003) in the context of M 87. Helical twists have been used to explain the structures in 3C 120 (Gómez et al. 1998, 2001) and it has been shown that superluminal motions along a curved trajectory can be explained by helical jet models, e.g., the radio source 3C 345 (Hardee 1987; Steffen et al. 1995). While some fitting of helically twisted structure has been performed, no detailed self-consistent models have been constructed. Modeling the emission from relativistic flows associated with the structures predicted by theory but including all relevant time delays can be used to provide the proper connection between the observations and the underlying properties of the outflows.

In this paper I present the basic behavior and appearance of a helical twist on a conical isothermal constant speed relativistic jet. I also show how the appearance of a helically twisted jet can be used to constrain the macroscopic properties of the jet and external fluid.

\section{Applicability of the Linearized HD Equations}

The development of helical jet structure on a relativistic jet can be obtained using a normal mode analysis of the linearized time dependent relativistic hydrodynamic equations. Previous studies indicate that helical patterns are likely to form in relativistic jets, can induce significant transverse motion without the development of shocks, and can produce asymmetries in Doppler boosted emission if jets are observed at viewing angles on the order of the beaming angle (Hardee 2000).

Non-relativistic numerical simulations indicate saturation of the normal wave modes of jet distortion in certain circumstances. The pinch and higher order normal wave modes grow only up to some saturation amplitude that does not lead to disruption of highly collimated jet flow (Hardee, Clarke \& Rosen 1997). Additionally, short wavelength helical twisting grows only up to some saturation amplitude that does not lead to disruption of highly collimated jet flow (Xu, Hardee \& Stone 2000). In these cases the normal modes remain within the "linear" regime. On the other hand, the simulations show that long wavelength helical twisting serves to disrupt ordered flow when the helical twist grows to non-linear amplitudes as a result of subsequent filamentation, mass entrainment and shock formation in the jet. Disruption by long wavelength helical twisting can be slowed by high Mach numbers, by strong magnetic fields, and by jet expansion (Rosen et al. 1999; Rosen \& Hardee 2000).

Within the "linear" non-destructive regime, helically twisted structures seen in numerical simulations have been successfully modeled by theory based on the non-relativistic MHD equations 
(Hardee \& Rosen 1999, 2002; Xu, Hardee \& Stone 2000) and the relativistic HD equations (Hardee et al. 1998, 2001; Agudo et al. 2001; Hardee \& Hughes 2003). This modeling showed that the theory correctly models the 3D pressure and velocity fields seen in non-relativistic and relativistic numerical simulations up to amplitudes that formally are much larger than linear, i.e., pressure fluctuations equal to the equilibrium pressure $\left(P=P_{0} \pm P_{1}\right.$ where $\left.P_{1} \leq P_{0}\right)$. Since numerical simulation results indicate jet disruption by asymmetric structures at non-linear amplitudes, highly collimated jets must be operating in the linear regime as otherwise they would be disrupted by asymmetric helical structures. Thus, I will assume that pressures and flow fields associated with observed jet structures can be modeled via the linear theory.

The polarization in jets, except in localized structures, is typically well below the maximum value of $\sim 70 \%$. This can be taken to mean that the magnetic field in jets beyond the acceleration region is not well organized. Disordered magnetic fields may provide a magnetic pressure but should not significantly influence the macroscopic normal mode structures predicted by the relativistic fluid equations.

\section{Modeling Helical Structures}

Declines in synchrotron intensity along superluminal jets indicate that $I \propto z_{\text {obs }}^{-1.3}$ (Homan et al. 2002) where $z_{\text {obs }}$ represents the observed separation distance from the core. Previous models of similar form typically have found power-law indices for VLBI jets (e.g., Walker, Benson \& Unwin (1987); Unwin \& Wehrle (1992); Xu et al. (2000)) between -1 and -2. VLA scale jets show similar power-law indices with increasing jet width, presumably proportional to the jet radius (Bridle \& Perley 1984). If we assume that this type of power-law decline scales with increasing jet radius in all cases, then this result strongly argues against adiabatic expansion. Thus, as a baseline case I will assume constant speed isothermal expansion. Isothermal expansion conserves energy flux for a constant jet speed. Formally, this means also that the magnetic pressure is negligible. It will also be assumed that the material surrounding the jet is isothermal. Such an assumption is reasonable if the jet is surrounded by a cocoon or lobe separating the jet from the ambient environment. Here the jet Mach number, $M_{j t} \equiv v_{j t} / a_{j t}$, remains constant and the external Mach number, $M_{e x} \equiv v_{j t} / a_{e x}$, also remains constant with the jet in pressure balance with the external cocoon or lobe medium.

\subsection{Helical Twisting: Moving Helical Patterns}

The linearized fluid equations show that a helical twist is Kelvin-Helmholtz unstable and a helical twist propagates at a wave speed dependent on jet speed, sound speeds, and on the frequency of the wave relative to a "resonant" or maximally unstable frequency, $\omega^{*}$. For sufficiently supersonic flow, the resonant frequency is $\omega^{*} R_{j t} / a_{e x} \sim 1.5$. The accompanying "resonant" wavelength depends on the wave speed.

At very low frequencies relative to the resonant frequency the wave speed is

$$
v_{w}=\frac{\gamma^{2} \eta}{1+\gamma^{2} \eta} v_{j t}
$$

and the wavelength

$$
\lambda(\omega)=\frac{\gamma^{2} \eta}{1+\gamma^{2} \eta}\left(\omega R_{j t} / v_{j t}\right)^{-1} R_{j t} .
$$

The spatial growth length is given by

$$
\ell(\omega)=\gamma \eta^{1 / 2}\left(\omega R_{j t} / v_{j t}\right)^{-1} R_{j t} .
$$

In the equations above $\gamma=\left(1-v_{j t}^{2} / c^{2}\right)^{-1 / 2}$ is the Lorentz factor and $\eta \equiv\left(a_{e x} / a_{j t}\right)^{2}$, where $a_{e x}$ and $a_{j t}$ are the sound speeds outside and inside the jet. The sound speed $a \equiv\left[\Gamma P_{0} /\left(\rho_{0}+[\Gamma /(\Gamma-\right.\right.$ 
1)] $\left.\left.P_{0} / c^{2}\right)\right]^{1 / 2}$, where $4 / 3 \leq \Gamma \leq 5 / 3$ is the adiabatic index. The density, $\rho_{0}$, and pressure, $P_{0}$, are measured in the proper fluid frames, and since pressure balance has been assumed $\eta$ is effectively an enthalpy ratio. For constant $\omega<<\omega^{*}$ the wave speed and growth length remain constant as the jet expands.

The wave speed changes as resonance is approached and at resonance the wave speed is (Hardee 2000)

$$
v_{w}^{*} \approx \frac{\gamma\left[M_{j t}^{2}-v_{j t}^{2} / c^{2}\right]^{1 / 2}}{\left[M_{e x}^{2}-v_{j t}^{2} / c^{2}\right]^{1 / 2}+\gamma\left[M_{j t}^{2}-v_{j t}^{2} / c^{2}\right]^{1 / 2}} v_{j t} .
$$

The corresponding "resonant" wavelength is

$$
\lambda^{*} \approx 3.75 \frac{\gamma\left[M_{j t}^{2}-v_{j t}^{2} / c^{2}\right]^{1 / 2} M_{e x}}{\left[M_{e x}^{2}-v_{j t}^{2} / c^{2}\right]^{1 / 2}+\gamma\left[M_{j t}^{2}-v_{j t}^{2} / c^{2}\right]^{1 / 2}} R_{j t},
$$

and the "resonant" spatial growth length is

$$
\ell^{*} \gtrsim \gamma M_{j t} R_{j t}
$$

This minimum growth length is typically greater than a straight extrapolation of the low frequency result to the resonant frequency. At very high frequencies and short wavelengths relative to resonance the wave speed becomes

$$
v_{w} \approx \frac{v_{j t}-a_{j t}}{1-a_{j t} v_{j t} / c^{2}}
$$

and the wave propagates like a backwards moving sound wave in the reference frame of the jet fluid. The growth rate is vanishingly small in the high frequency and short wavelength limit.

The analytic expressions can be used to provide limiting estimates of wave speed, wavelength and amplitude growth to be expected of a fixed frequency wave along an expanding jet. However, detailed development requires numerical solutions to the dispersion relation describing helical wave propagation and growth. Solutions to this dispersion relation for a jet speed of $\beta_{j t} \equiv v_{j t} / c=0.99$, an adiabatic index of $\Gamma=13 / 9$ appropriate to a mixture of hot leptonic and cold baryonic material (Synge 1957), and representative choice of sound speeds are shown in Figure 1. Panels in Figure 1 have been arranged so that the resonant frequency normalized by the jet speed and jet radius, $\omega^{*} R_{j t} / v_{j t}$, remains nearly constant along a diagonal direction. Similar wave speed and wavelength behavior as a function of $\omega R_{j t} / v_{j t}$ occurs along the vertical direction. For a sufficiently relativistic jet, behavior is governed entirely by the Lorentz factor and sound speeds.

Figure 1 also shows the intrinsic and apparent wave speeds and wavelengths associated with the dispersion relation solutions where I have chosen a jet viewing angle of $\theta_{0}=14^{\circ}$. With this assumed viewing angle and a flow Lorentz factor of $\gamma \equiv\left[1-\beta_{j t}^{2}\right]^{-1 / 2} \sim 7$, the maximum apparent superluminal speed is $\beta_{\max }^{\text {obs }} \approx 6$. Note that at this viewing angle subluminal apparent motions occur for wave speeds $\beta_{w} \equiv v_{w} / c<0.78$ with $\gamma_{w}<1.6$. The apparent helical wavelength in the observer frame is related to the intrinsic wavelength by $\lambda^{o b s}=\beta_{w}^{o b s} \lambda$ and the intrinsic wavelength can be greater or less than the observed wavelength depending on wave speed and viewing angle. For our viewing angle, a wave speed equal to the flow speed implies $\lambda^{o b s} \sim 6 \lambda$ but when $\beta_{w}<0.78$ $\lambda^{o b s}<\lambda$. When sound and jet speeds remain constant, a helical wave of fixed frequency, $\omega$, remains at constant wavelength in the long and short wavelength regimes where the wave speed is constant.

Even with constant sound and jet speeds, a helical wave of fixed frequency changes propagation speed and wavelength as $\omega R_{j t} / a_{e x}$ moves from the low frequency to the high frequency regime as a 

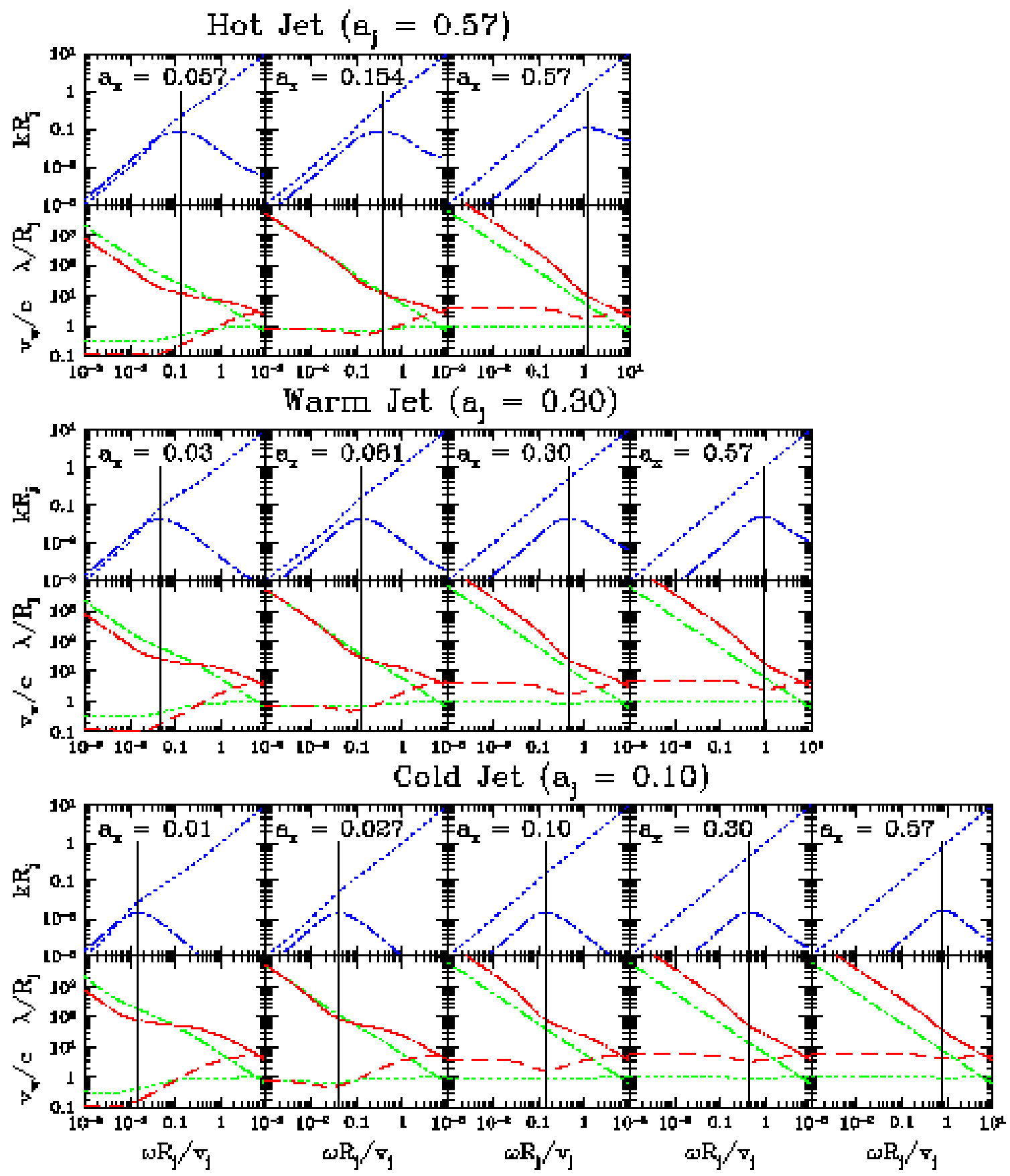

Fig. 1. - Solutions to the dispersion relation $k R_{j t}$ as a function of $\omega R_{j t} / v_{j t}$ for a helical wave. Dotted (Dash-dot) lines indicate the real (imaginary) part of the wavenumber, and the sound speed is indicated in units of $c$. The intrinsic (dotted line) and apparent (dashed line) wave speeds and intrinsic (short dash-dot line) and apparent (long dash-dot line) wavelengths are shown immediately below the appropriate dispersion relation solutions. The vertical line indicates the location of the resonant frequency. 
result of increase in $R_{j t}$. Change in the observed wavelength is amplified relative to change in the intrinsic wavelength, $\lambda(z)=\left[\beta_{w}(z) / \beta_{w}\left(z_{0}\right)\right] \lambda\left(z_{0}\right)$, with

$$
\lambda^{o b s}\left(z \sin \theta_{0}\right)=\frac{\sin \theta_{0}}{1-\beta_{w}(z) \cos \theta_{0}} \frac{\beta_{w}(z)}{\beta_{w}\left(z_{0}\right)} \lambda\left(z_{0}\right) .
$$

In equation (4), $z$ is the intrinsic distance along the jet axis where $z_{o b s} \equiv z \sin \theta_{0}$ is the observed distance. The analytical and numerical solutions to the dispersion relation indicate that significant change in the wavelength can occur when $a_{e x} / a_{j t}<1$. Much reduced variation occurs in observed wave speed when $a_{e x} / a_{j t}>1$. In particular, note that $a_{e x} / a_{j t} \ll 1$ implies $\beta_{w} \ll 1$ when $\omega R_{j t} / v_{j t} \ll$ $\omega^{*} R_{j t} / v_{j t}$ and $\beta_{w} \lesssim 1$ when $\omega R_{j t} / v_{j t} \gg \omega^{*} R_{j t} / v_{j t}$ (leftmost panels in Figure 1). The resulting broad plateau seen in $\lambda^{o b s} / R_{j t}$ implies that a fixed frequency helical wave propagating along an expanding jet will appear to increase in wavelength approximately proportional to $R_{j t}$ over the corresponding normalized frequency range, and will transition from less than to greater than the intrinsic wavelength. The corresponding normalized frequency range allows this behavior to be observed over up to two orders of magnitude of jet expansion. This wavelength change would be accompanied by a similar large change in the observed wave speed.

As a helical twist is Kelvin-Helmholtz unstable, the displacement amplitude of the jet surface grows according to

$$
A=A_{0} \exp \left[\int_{z_{0}}^{z} \ell(z)^{-1} d z\right],
$$

where $A_{0}$ is the displacement amplitude at $z_{0}$. However, the magnitude of accompanying velocity and pressure fluctuations grows according to $A(z) / R_{j t}(z)$, and for constant jet expansion

$$
\frac{A(z)}{R_{j t}(z)}=\frac{A_{0}}{R_{0}} \frac{\exp \left[\int_{z_{0}}^{z} \ell(z)^{-1} d z\right]}{\left[1+\left(z-z_{0}\right) \psi / R_{0}\right]},
$$

where $R_{0}$ is the jet radius at $z_{0}$ and $R_{j t}=R_{0}+\left(z-z_{0}\right) \psi$. In the absence of non-linear stabilizing effects, $\ell(z)$ from equation (1c) in the low frequency limit is constant, begins to increase as resonance is approached and then rapidly increases at higher frequencies. For a jet to both exhibit helical twisting and remain collimated the amplitude must grow sufficiently rapidly but not so rapidly that large amplitudes are reached while the wave is in the low frequency regime. In order that the helical twist not be damped in the low frequency regime we must have that $A(z) / R_{j t}(z) \geq A_{0} / R_{0}$. Scaling of the growth length proportional to the wavelength in the low frequency regime coupled to a minimum in the growth length at $\omega^{*} R_{j t} / a_{e x}$ means that the maximum in $A(z) / R_{j t}(z)$ occurs when $\omega R_{j t} / a_{e x}>\omega^{*} R_{j t} / a_{e x}$. Note however that constant pressure and velocity fluctuations can be maintained for decreasing $A(z) / R_{j t}(z)$ when $\omega R_{j t} / a_{e x}>>\omega^{*} R_{j t} / a_{e x}$ (see $\S 3.2$ ).

Theoretically, these basic properties allow estimation of jet parameters, i.e., sound speeds for a given Lorentz factor and/or viewing angle without detailed modeling of the intensity structure. The viewing angle and flow Lorentz factor are constrained by the highest observed superluminal speed, assumed related to the flow speed. Lack of damping sets an upper limit to $\gamma M_{j t}$. A constant wavelength indicates a helical pattern in the low or high frequency limits. In the low frequency limit this sets a value for $\gamma a_{e x} / a_{j t}$. In the high frequency limit this sets a value for $a_{j t}$. If the helical wavelength appears to scale approximately with the jet radius over some expansion range, then this can be used to set an upper limit to $\gamma a_{e x} / a_{j t}$ and lower limit to $a_{j t}$ from lower and upper limits to the wave speed, respectively.

\subsection{Helical Twisting: Decoupling the Pattern and Fluid Flow}

Examination of the fluid flow accompanying a growing helical twist on an expanding jet can be accomplished by constructing 3D theoretical data cubes using the expressions giving the pressure 
and velocity structure associated with the normal modes (Hardee et al. 1998; Hardee 2000). Formally, these expressions assume a cylindrical jet. However, conical jet expansion at small opening angle does not significantly modify local solutions to the dispersion relation (Hardee 1984). In what follows I adopt a jet half opening angle of $\psi=0.025$ radian that is less than the relativistic Mach angle, $\sim\left(\gamma M_{j t}\right)^{-1}$, for the two cases to be considered. The flow and pressure field accompanying a helical twist can be evaluated quantitatively by taking 1-D cuts through the theoretically generated 3 -D data cubes at constant values of $x / R_{j t}$ as illustrated in Figure 2. For 1-D cuts in the x-z plane $v_{x}$ and $v_{y}$ are radial and azimuthal velocity components in cylindrical coordinates.

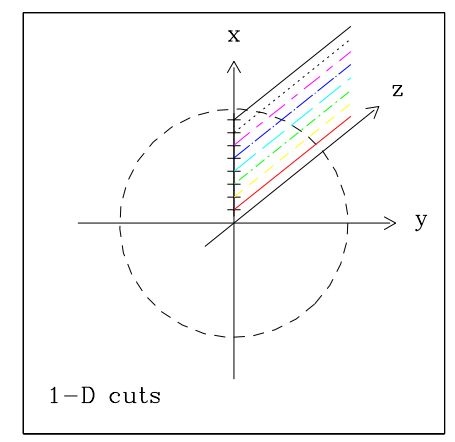

Fig. 2.- 1-D cuts at $\mathrm{x} / \mathrm{R}=0.11$ (solid line), 0.22 (short dash line) 0.33 (dot-short dash line), 0.44 (long dash line), 0.55 (dot-long dash line), 0.66 (short \& long dash line), 0.77 (dotted line), \& 0.88 (solid line). Here we look downstream and $z$ is into the page.

The evolution of the pressure and velocity components of frequencies $\omega R_{0} / v_{j t}=0.01,0.03,0.1$ for a "hot" jet $\left(a_{j t}=0.57\right)$ with $a_{e x}=0.057$ and for a "warm" jet $\left(a_{j t}=0.30\right)$ with $a_{e x}=0.081$ is shown in Figure 3. These two cases, see Figure 1, have been chosen because the resonant frequency and intrinsic wavelengths are comparable but sound speeds are significantly different. As a result, the wave speed on the warm jet is significantly larger and growth rate on the warm jet is significantly reduced compared to the hot jet. In order to obtain similar growth with "saturation" amplitude occurring at a frequency $\omega>>\omega^{*}$, an initial amplitude of $A_{0} / R_{0}=0.0007$ and 0.07 is chosen for hot and warm jets, respectively. The large difference in initial amplitudes indicates an extreme sensitivity of the growth rate to the jet sound speed, i.e., decrease in jet sound speed by a factor 2 has required an initial amplitude increase by a factor 100 . In fact $A / R_{j t}$ initially declines for the low frequency wave on the warm jet. Saturation is imposed when pressure fluctuations reach the maximum allowed by the linear analysis. Saturation was imposed at the medium frequency on hot and warm jets and at the high frequency on the warm jet. It was found that saturation in pressure fluctuations was maintained by imposing a decrease in the displacement amplitude of the form $A=A(z) \times\left(2 k_{R}^{*} / k_{R}\right)^{n}$ when $\left(2 k_{R}^{*} / k_{R}\right) \leq 1$, where $k_{R}$ is the real part of the wavenumber, $k_{R}^{*}$ corresponds to resonance and $n \leq 2$.

The velocity fluctuations on the warm jet are $\approx 55 \%$ of that on the hot jet for a given pressure fluctuation and the difference reflects the decrease in sound speed and increase in wave speed on the warm jet. Note that jet expansion is indicated by the spread in radial velocities across the jet. While not readily apparent from these plots, the jet attempts to move bodily around the central axis. See, for example, the velocity vectors in Figures 4 \& 7 in Hardee et al. (2001) that indicate uniform sideways motion of the jet fluid across a jet cross section. Here sideways motion is impeded at the jet surface by the external fluid. The "heavier" external fluid surrounding the hot jet impedes the surface motion, relative to motion of the jet interior, more than is the case for the warm jet. Note that shorter intrinsic wavelengths are associated with higher wave speed in these two cases. For a higher wave speed the fluid does not need to move as rapidly in the transverse 

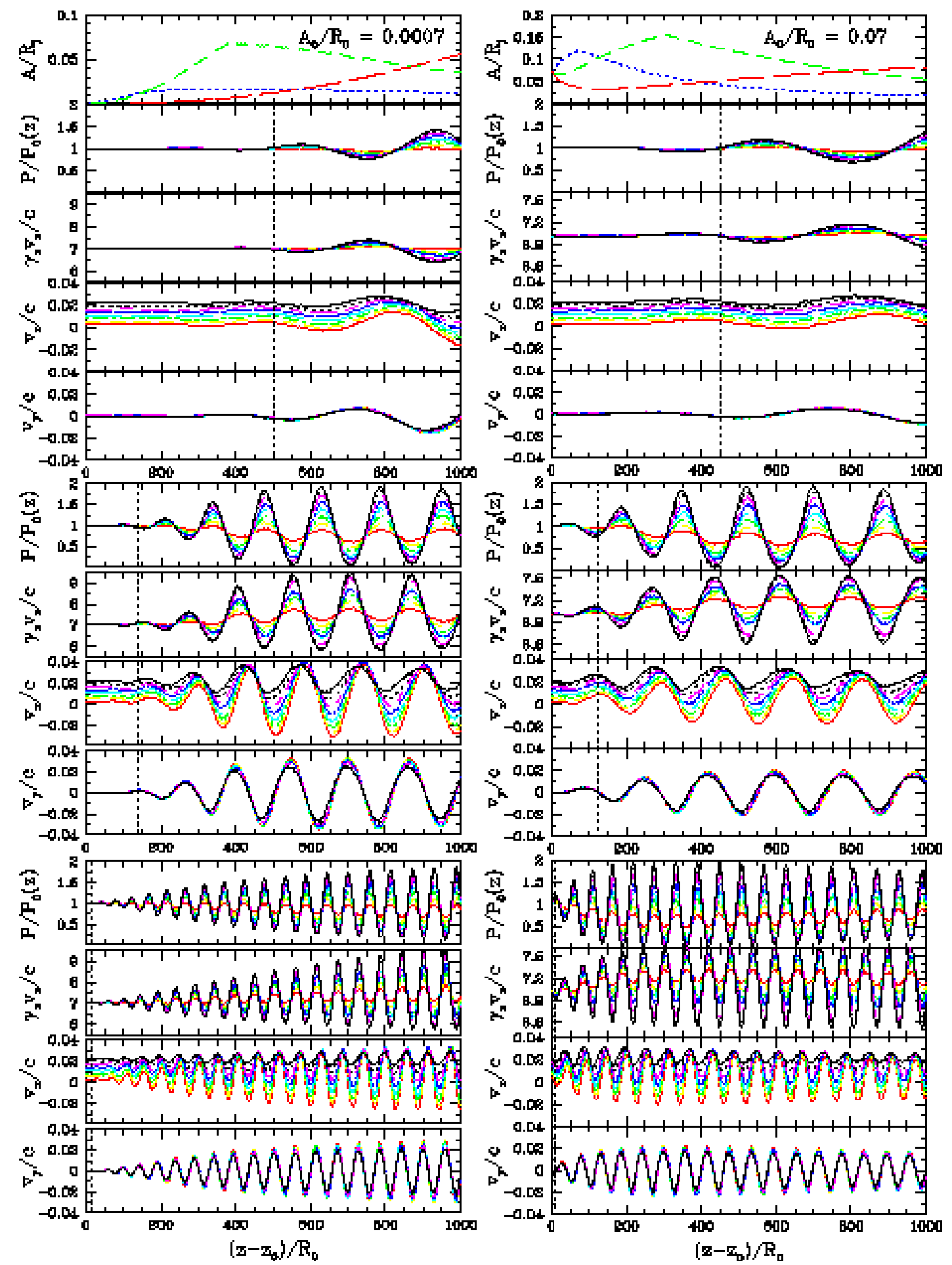

Fig. 3. - The leftmost (rightmost) set of panels is for the hot (warm) jet. The topmost panel shows the amplitude growth of $A / R_{j t}$ as a function of distance for low (long dash line), medium (short dash line) and high (dotted line) frequencies. Three sets of panels below show the normalized pressure $P / P_{0}(z)$, and velocity components $\gamma_{z} v_{z} / c, v_{x} / c$ and $v_{y} / c$ for low (top set), medium (middle set) and high (bottom set) frequency. The dotted vertical line in the panels indicates the position at which the wave frequency equals the local resonant frequency. 
direction to circulate through the helical pattern. This effect tends to cancel the higher circulation speed otherwise required by a shorter wavelength helical pattern.

The appearance of the high pressure ridge corresponding to the low, medium and high frequency fluctuations shown in Figure 3 is shown in Figure 4. The images in Figure 4 are line of sight integrations through 3-D pressure data cubes. In constructing the data cubes it is assumed that the pressure on the unperturbed conical jet declines as $P_{0}(z) \propto R_{j t}(z)^{-2}$. Only one set of images

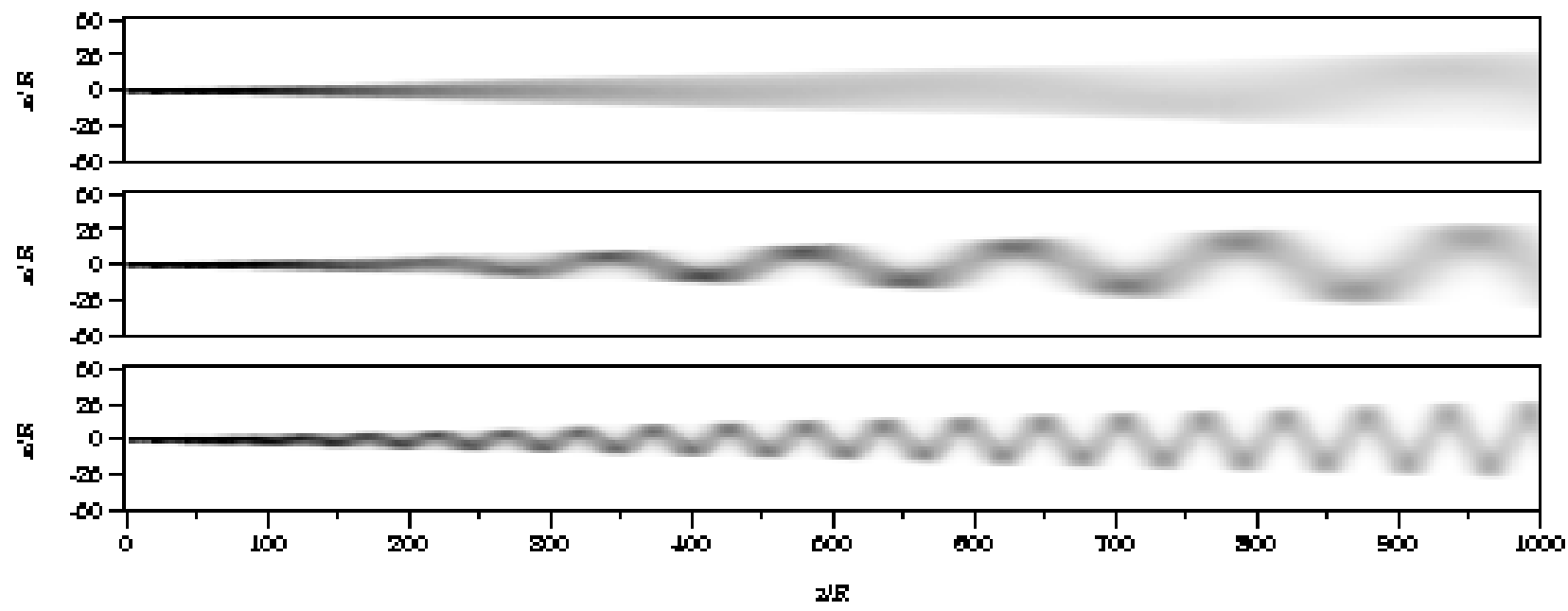

Fig. 4. - Line of sight integration through 3-D pressure data cubes. The three panels show the appearance of the helical pressure ridge that accompanies the low (top), medium (middle) and high (bottom) frequency hot and warm jet fluctuations shown in Figure 3.

for low, medium and high frequencies is shown as hot and warm jets appeared nearly identical. For all frequencies the surface displacement of the jet is small and the images show that the high pressure ridge appears to lie within the conical jet's surface. In general, the jet fluid does not follow the path of the high pressure ridge. If we define the pitch angle of the flow by $\tan \theta_{f}=\left(v_{\perp} / v_{z}\right)$, then $\tan \theta_{f} \leq 0.035 \& 0.02$ for saturation amplitudes on the hot and warm jets, respectively, where $v_{\perp} / v_{z} \sim\left|v_{x} / c\right|_{\max } \sim\left|v_{y} / c\right|_{\max }$ from Figure 3. This maximum flow pitch angle is on the order of half the relativistic Mach angle, i.e., $\theta_{f} \leq \theta_{\text {rel }} / 2=\left(2 \gamma M_{j t}\right)^{-1}$. The helical pitch of the high pressure ridge defined by $\tan \theta_{h}=2 \pi R_{j t} / \lambda$ varies from $0.04<\tan \theta_{h}<1$ at the medium frequency and $0.11<\tan \theta_{h}<3$ at the high frequency as $\left.0 \leq\left(z-z_{0}\right) / R_{0}\right) \leq 1000$ on both hot and warm jets. As a result of the decoupling between the pattern and fluid flow, the variation in flow angle, $\theta=\theta_{0} \pm \Delta \theta$ where $\Delta \theta \lesssim \theta_{f}$, is always less than would be inferred from the helical pitch of the high pressure ridge.

\subsection{Helical Twisting: Observing Helical Patterns}

The brightest radio emission is expected to trace the path of the high pressure ridge. In constructing line of sight images it will be assumed that a pseudo-synchrotron emissivity at fixed frequency can be written as

$$
\epsilon_{\nu} \propto n_{j t}^{1-2 \alpha} p_{j t}^{2 \alpha}\left(B \sin \theta_{B}\right)^{1+\alpha} D^{2+\alpha}
$$

where $\theta_{B}$ is the angle of the magnetic field to the line of sight and $D \equiv[\gamma(1-\beta \cos \theta)]^{-1}$ is the Doppler boost factor for fluid flowing with speed $\beta$ at angle $\theta$ to the line of sight. I have used $\alpha=0.5$ where $\nu^{-\alpha}$ and set $\theta_{B}=$ constant. This form for the pseudo-synchrotron emissivity is necessary when the synchrotron emitting particles are not explicitly tracked (Clarke, Norman \& Burns 1989). 
That pseudo-synchrotron intensities provide reasonable images of internal jet structures is indicated by the work of Jones, Ryu \& Engel (1999). Along the conical constant velocity isothermal jet the particle number density and pressure are assumed to decline $\propto R_{j t}^{-2}$. The magnetic field strength is assumed to decline as $B \propto n_{j t}^{2 / 3} \propto R_{j t}^{-4 / 3}$ appropriate to a disordered magnetic field (Heinz \& Begelman 2000).

\subsubsection{Individual Frequencies}

Line of sight pseudo-synchrotron images corresponding to the low, medium and high frequency hot and warm jet cases shown in Figures $3 \& 4$ are shown in Figure 5. In these images all wave motion, light travel time and Doppler boosting effects appropriate to a jet at a $\theta_{0}=14^{\circ}$ viewing angle have been incorporated, and the apparent opening angle of the conical jet is $\approx 12^{\circ}$. The $x, y, z$ coordinate system is defined by the jet with $x$ in the sky plane and $z$ down the jet axis with the hypothetical observer located in the $y-z$ plane. Comparison between Figure 4 (similar to
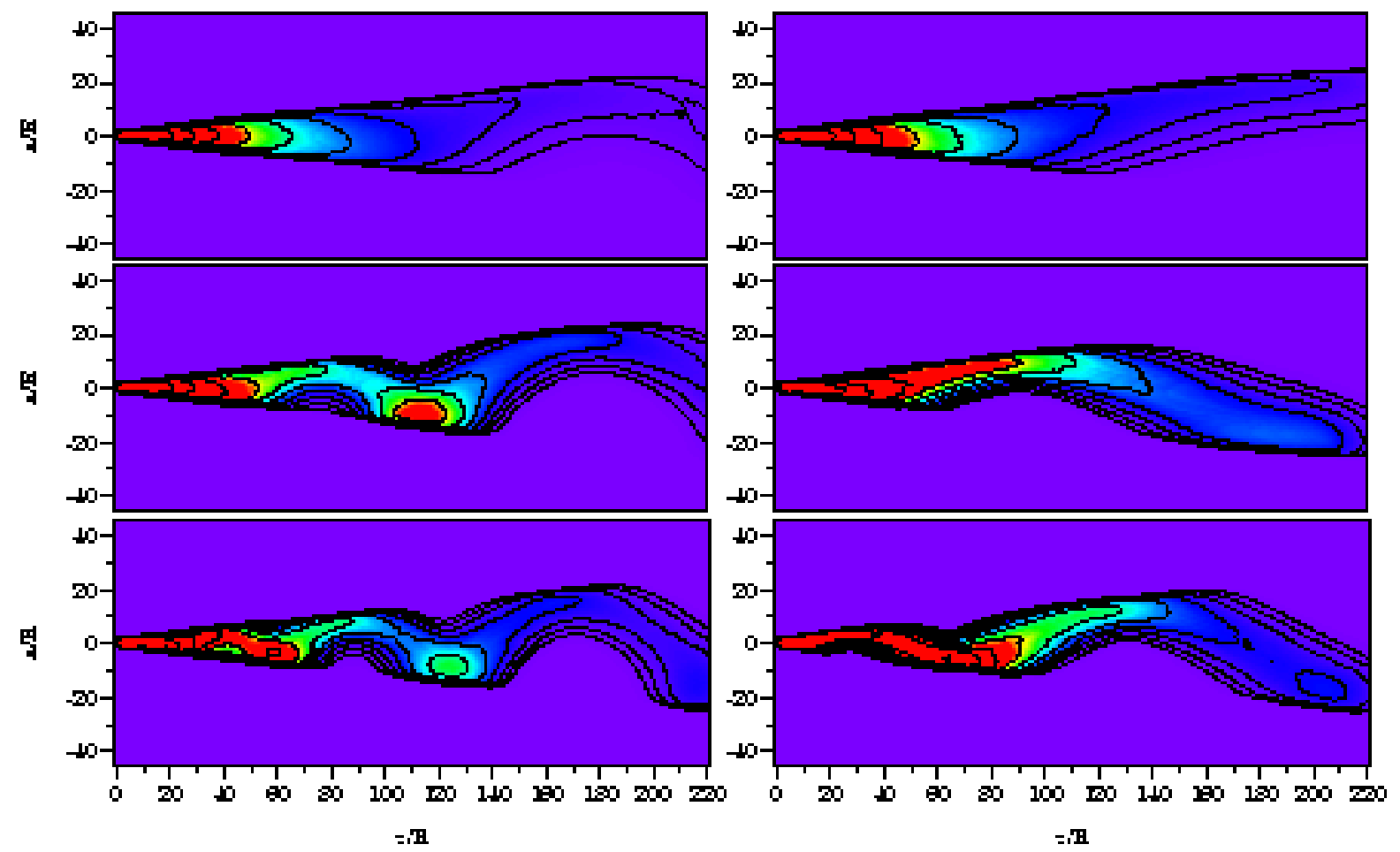

Fig. 5.- Pseudo-synchrotron intensity images for low (top), medium (middle) and high (bottom) frequency helical waves on hot (left panels) and warm (right panels) jets at a viewing angle of $\theta=14^{\circ}$. The contours levels are in factors of 2 .

a pseudo-synchrotron intensity image of the jet in the plane of the sky) and Figure 5 provides a striking example of the effect of wave motion and the accompanying light travel time effects on the apparent wavelength. On the hot jet the apparent wavelength varies from less than to greater than the intrinsic wavelength from low to high frequencies, respectively. On the warm jet the apparent wavelength varies from comparable to much greater than the intrinsic wavelength from low to high frequencies, respectively. At all three frequencies the longer apparent wavelength on the warm jet is the result of faster wave motion. The reduced wave speed on the hot jet and accompanying reduced apparent wavelength are sufficient to have produced intensity knots. 
Increase in the apparent wavelength with $z$ at medium and high frequencies on the hot jet and at high frequency on the warm jet results from increase in the intrinsic wave speed.

The existence of intensity knots and ratio of brightnesses between knot and interknot regions can be used to constrain helical flow models, e.g., Walker et al. (2001). For the hot jet medium and high frequency helical twists, the intensity ratio between knot and interknot regions is $\approx 4$. In general, such intensity knots are the result of a combination of helical twist projection and Doppler boosting effects where the magnitude and direction of flow is modified by high and low pressure regions. For nearly constant Lorentz factor, the Doppler boost factor is approximately given by

$$
D=D_{0} \pm \Delta D \approx D_{0}\left[1 \pm \frac{\beta_{0} \theta_{0}}{\left(1-\beta_{0} \cos \theta_{0}\right)} \Delta \theta\right]
$$

where $\Delta \theta \lesssim \theta_{f}$. Along the hot conical jet with a saturated helical twist at $\omega>\omega^{*}$ the near side can be boosted relative to the far side and the bottom can be boosted relative to the top with $\theta_{f} \leq 0.035$ radian. Thus, with $D_{0} \sim 3.6$ we expect $\Delta D \sim 0.2 D_{0} \lesssim 0.7$ and a maximum variation in $D^{2.5}$ of $\sim 2.5$. About half of the intensity variation results from simple projection effects and half from Doppler boosting effects. The higher wave speed associated with the warm jet has reduced transverse motion and Doppler boosting contrast, and the longer apparent wavelength has reduced projection effects such that intensity knots are almost eliminated.

\subsubsection{Multiple Frequencies}

For a single frequency excited by precession of the central engine the behavior of a helical twist is predictable over many orders of magnitude of jet expansion. Difficulties arise if multiple frequencies are excited. At any location along the "linear" expanding jet the frequency that has grown the most will be above the local resonant frequency. This frequency reaches saturation or some lesser amplitude depending on the initial amplitude and growth rate, but could be overwhelmed by a helical wave at lower frequency that continues to grow and so on. Since the resonant wavelength scales with the jet radius, we would expect to see approximate scaling with the jet radius, albeit with jumps in wavelength.

Perhaps the largest uncertainty in dealing with multiple frequencies lies in the interaction between multiple frequencies associated with the same wave mode. Numerical simulations have shown that the faster growing higher order normal modes (elliptical, triangular etc.) do not slow the growth of a helical mode wave and decline in amplitude as the longer wavelength helical twist grows (Hardee, Clarke \& Rosen 1997). Another simulation has shown that pinch mode waves show relatively abrupt change to longer wavelength on a conically expanding axisymmetric jet (Agudo et al. 2001). Therefore I follow the simple assumption that a high frequency saturated helical wave will decline in amplitude as a lower frequency helical wave grows as specified by the linear theory. Specifically I consider the hot jet with a combination of low and medium frequency waves and with a combination of low and high frequency waves. I choose the hot jet as the variation in wave speed from low to high frequencies is more extreme than on the warm jet. Jet speed and opening angle remain the same as the previous single frequency models.

The results of this study are shown in Figure 6 . Figure 6 contains plots of $A / R_{j t}$ for each wave, 1-D cuts of the combined wave pressure and velocity fluctuations, and pseudo-synchrotron intensity images for the jet in the plane of the sky and for the jet at a viewing angle of $14^{\circ}$. The combined pressure and velocity fluctuations are the result of a linear combination of fluid displacements associated with the individual waves from which the velocity components and pressure are computed. Note that the pressure is not a linear combination of pressures associated with the individual waves. Combined low and medium frequency waves grow from an initial $A_{0} / R_{0}=0.0007$. Saturation is reached for low and medium frequency waves when the individual wave frequency is somewhat larger than twice the local resonant frequency. The linear combination of the two 
waves remains saturated if the amplitude of the individual waves behaves like $A \times\left(2 k^{*} / k\right)^{3}$ when $\left(2 k^{*} / k\right) \leq 1$. Previously, I found $\left(2 k^{*} / k\right)^{2}$ maintained saturation for the medium frequency wave alone. For combined low and high frequency waves the high frequency wave grows from an initial $A_{0} / R_{0}=0.001$ in order to reach saturation before declining in amplitude like $A \times\left(8 k^{*} / k\right)^{3}$ when $\left(8 k^{*} / k\right)<1$.
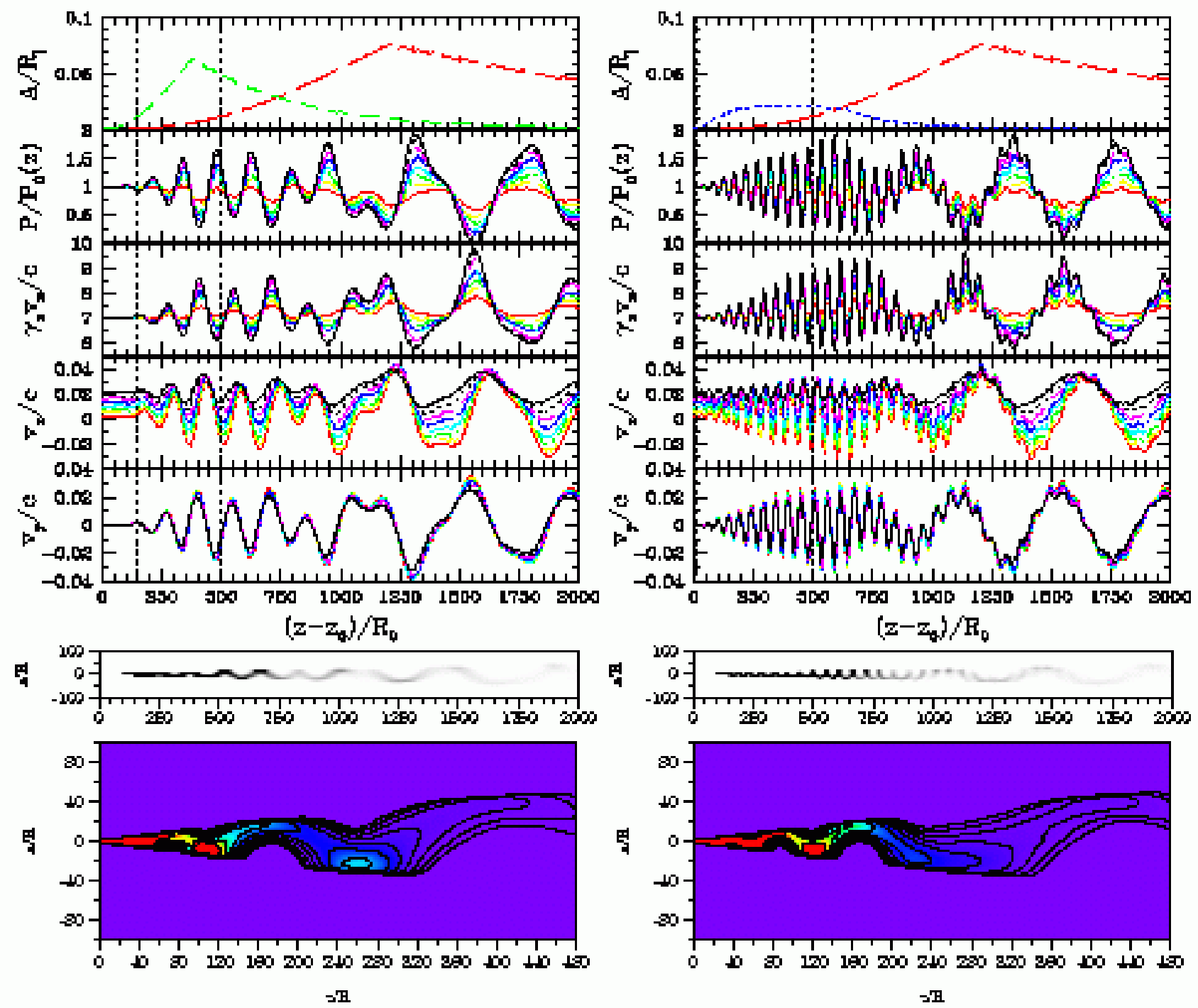

Fig. 6. - The leftmost (rightmost) set of panels is for the low/medium (low/high) frequency combinations. The topmost panel shows the amplitude growth of $A / R_{j t}$ as a function of distance for low (long dash line), medium (short dash line) and high (dotted line) frequencies. Panels below show the pressure and velocity components as in Figure 3. The two dotted vertical lines in the panels indicate the position at which the higher and lower wave frequency equals the local resonant frequency. Below these panels pseudo-synchrotron intensity images for low $/$ medium and low/high frequency combinations for the jet in the plane of the sky and for the jet at a $14^{\circ}$ viewing angle are shown. The contour levels are in factors of 2 .

The rapid motion of the high frequency wave leads to significant light travel time effects across the jet diameter, visible as cusps at the top of the jet, for the image corresponding to the jet in the plane of the sky. At the $14^{\circ}$ viewing angle relatively smooth scaling of the helical wavelength 
with jet radius (low plus medium frequency wave) or more abrupt jump in helical wavelength (low plus high frequency wave) is observed. Knot motion can be complex for multiple frequency waves. For the low and medium frequency combination the knot at $z / R \sim 110,\left(z-z_{0}\right) / R_{0} \sim 450$, will move with $\beta_{w}^{o b s} \sim 0.35$. The knot at $z / R \sim 260,\left(z-z_{0}\right) / R_{0} \sim 1070$, is a combined feature and could show a component from the medium frequency wave with $\beta_{w}^{o b s} \sim 1$ moving through a slower $\beta_{w}^{o b s} \sim 0.3$ feature associated with the low frequency wave. For the low and high frequency case the knot at $z / R \sim 120\left[\left(z-z_{0}\right) / R_{0} \sim 500\right]$ will move with $\beta_{w}^{o b s} \sim 2$ while the low frequency wave moves with $\beta_{w}^{o b s}<0.3$. At an apparent distance of $z / R=500\left[\left(z-z_{0}\right) / R_{0} \sim 2100\right]$ the low frequency wave moves with $\beta_{w}^{\text {obs }} \sim 0.6$. Recall that knots moving with the flow would appear to move at $\beta_{f}^{o b s} \sim 6$ and the maximum possible wave speed found using equation (3) is $\left.\beta_{w}^{o b s}\right|_{\max }=3.7$.

\section{The inner $3 \mathrm{C} 120$ jet as an example}

The radio source 3C 120 exhibits structure on scales from under a parsec to hundreds of kiloparsecs (Walker, Benson \& Unwin 1987). The galaxy has a redshift $z=0.033$ (Baldwin et al. 1980), and following Gómez et al. (2001) I let $H_{o}=h 100 \mathrm{~km} \mathrm{~s}^{-1} \mathrm{Mpc}^{-1}$ with $h=0.65$ and there are $\approx 0.70 h_{65}^{-1} \mathrm{pc} \mathrm{mas}^{-1}$. Recent high frequency VLBI observations at 86, 43 and $22 \mathrm{GHz}$ (Gómez et al. 2000, 2001) have produced high resolution images of the inner jet. Components $s, r, m, o, h \& d$, shown here in Figure 7 (see also Gómez et al. (2001) Fig. 1), located successively farther from the core have motions $\approx 0.27,0.40,0.49,1.83,1.75 \& 1.71$ mas yr$^{-1}$ respectively, where 1 mas $\mathrm{yr}^{-1}=2.34 h_{65}^{-1} \mathrm{c}$. The separation of these components increases from $s$ to $o$ with $o$ at $\approx 2$ mas from the core.

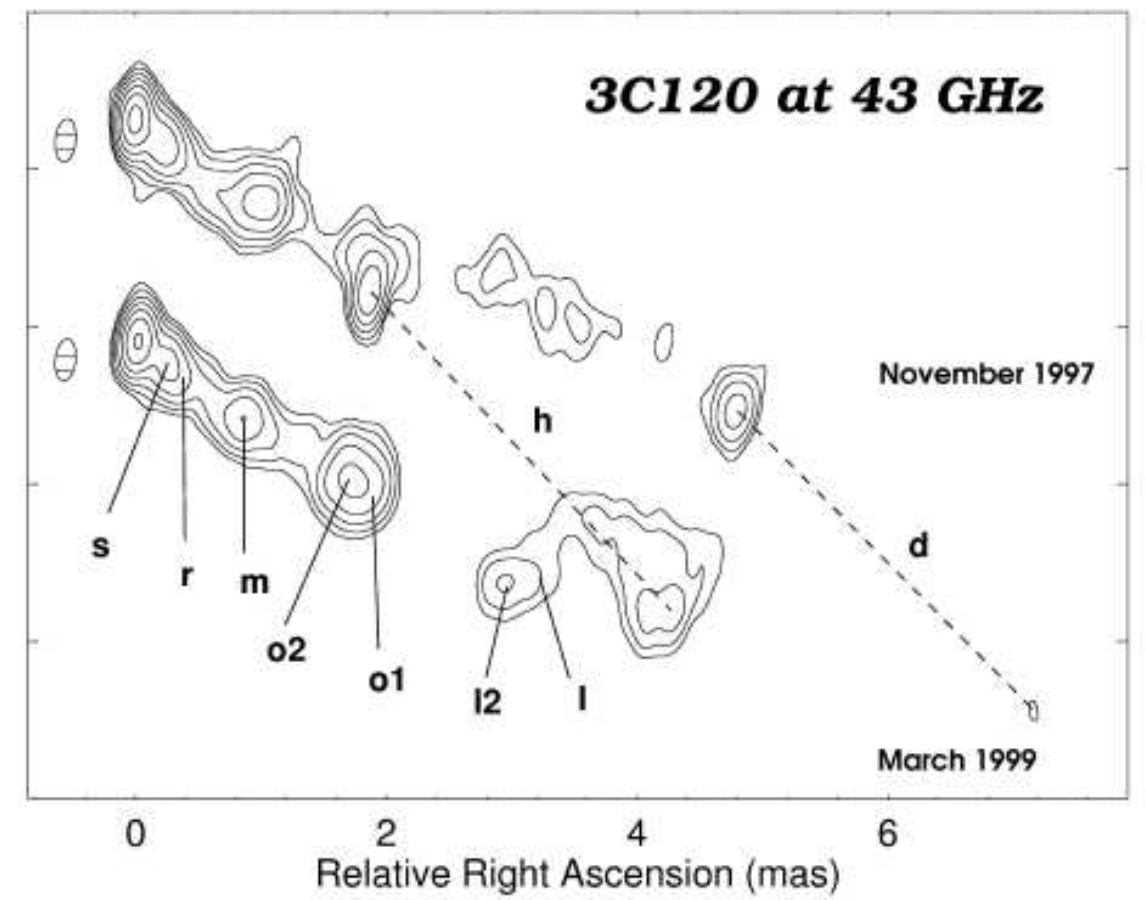

Fig. 7.- Synchrotron intensity images at $43 \mathrm{GHz}$ of the $3 \mathrm{C} 120$ jet at two epochs. Component o in the text corresponds to $\mathrm{o} 2 \& \mathrm{o} 1$ in the image. Contour levels are in factors of 2 . Image provided by Jose-Luis Gómez. 
Gómez et al. (1998) suggested that components $h$ and $d$ located at $\sim 2$ mas and $\sim 5$ mas from the core in 43 and $22 \mathrm{GHz}$ images in Gómez, Marscher \& Alberdi (1999) (Figure 1) were the result of a projected helical path and experienced a different evolution from other components that formed a curved path between and beyond these two components. The relatively constant spacing and motion along with "saturated" component intensity structure beyond 2 mas indicates that these components represent a helical twist in the high frequency regime. In Gómez et al. (2001) fast moving components between $o$ and $h$ (e.g., l \& 12 in Fig. 7) show motions from 2.17

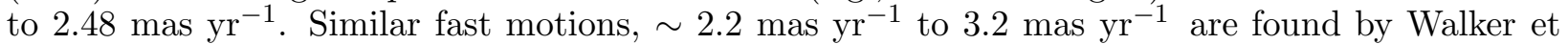
al. (2001) at $5 \mathrm{GHz}$ out to 14 mas from the core. An average apparent motion associated with

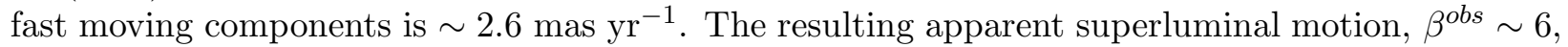
constrains the viewing angle to less than $19^{\circ}$ and a minimum fast moving component speed of $\beta=0.986$ is obtained at a viewing angle $\theta=9.6^{\circ}$. It is likely that the average speed of the faster moving components is indicative of the flow speed, and the motion of components $h$ and $d$ is indicative of helical wave motion in the high frequency regime.

Components $s-m$ have been interpreted as pinch structures (Gómez et al. 2001) triggered by passage down the jet of a shock associated with component $o$. Such an effect was observed by Agudo et al. (2001) in numerical simulation. In what follows I will assume instead that these newer components are also associated with a helical wave and that the average apparent speed, $\beta^{\text {obs }}=4.14$ c, of components $o, h \& d$ represents motion of the helical wave in the high frequency regime. The apparent wavelength in the high frequency regime is then given by the spacing, $2.5-3$ mas, between components $o, h \& d$. The apparent increasing speed of components $s, r, m \& o$ is associated with a change in helical wave speed as a wave of single frequency makes a transition from the low to high frequency regime by virtue of jet expansion. The apparent speed of component $s$ nearest to the core, $\beta^{\text {obs }}=0.63$, is assumed to represent motion of the helical wave in the low frequency regime.

In what follows I will consider a single viewing angle of $9.6^{\circ}$. Obviously a range of angles and computation of synchrotron intensity is needed to constrain the viewing angle but this modeling is beyond the scope of the present work. With an observed jet opening angle of $\sim 12^{\circ}$, the true half-opening angle becomes $\approx 1^{\circ}$. At the assumed viewing angle the true wave speed changes from low to high frequency regimes as $0.80 \leq \beta_{w} \leq 0.974$ and the true wavelength increases by a factor $1.22=0.974 / 0.80$. The observed component spacing associated with the apparent helical wavelength would increase according to equation (4) by a factor 6.6 from component $s$ to component $o$, or from, say, 0.4 to 2.6 mas consistent with the observed component spacing. The corresponding true wavelength, $\lambda$, varies from $0.35 \mathrm{pc}$ near the core to $0.43 \mathrm{pc}$ at $8.4 \mathrm{pc}$ ( 2 mas observed) from the core where the jet radius, $R_{j t}$, is $\approx 0.15 \mathrm{pc}(\approx 0.21 \mathrm{mas})$, and $\lambda \sim 3 R_{j t}$.

A wave speed of $\beta_{w}=0.974$ in the high frequency regime and flow speed $\beta=0.986$ implies a jet sound speed $a_{j t}=0.3 c$ according to equation (3). A wave speed of $\beta_{w}=0.80$ in the low frequency regime implies $a_{e x} / a_{j t}=0.35$ and $a_{e x}=0.1 c$ from equation (1a). These derived parameters are very similar to those used for the "warm" jet case shown in the previous sections. The corresponding dispersion relation solution panel in Figure $1\left(a_{e x}=0.081 c\right)$ clearly shows that $\lambda \sim 3 R_{j t}$ is in the high frequency regime as assumed. The wavelength and wave speed inferred from the observations imply a frequency $\omega R_{j t} / a_{e x} \sim 60\left(\omega R_{j t} / v_{j t} \sim 6\right)$ at component $o(z \sim 8.4 \mathrm{pc})$.

On a strictly isothermal jet, the dispersion relation solution implies a jet expansion by a factor $\approx 40$ beginning at $z \sim 0.21 \mathrm{pc}$ to achieve the observed acceleration in wave speed. This position is inside component $s$, has $\omega R_{j t} / a_{e x} \sim 1.5$ and implies an initial excitation at about the resonant frequency. In this case the speed of component $s$ may more closely represent the wave speed at resonance. Unfortunately the estimated wave speed at resonance given by equation (2a) is insufficiently accurate for our purposes and, in general, a numerically found value must be used. For the present parameters the wave speed at resonance is slightly less than in the low frequency regime and thus $a_{e x} \gtrsim 0.1 \mathrm{c}$ would be implied. Note that the inferred jet parameters allow for an apparent 
faster motion of components closer to the core, if the helical path could be followed/existed inside component $s$.

The amplitude growth curve for the high frequency helical wave on a "warm" jet (Figure 3 top right panel) gives a good indication of expected wave growth for a helical wave beyond the resonant location independent of the exact location or mechanism for the initial perturbation. Here we have a resonant location $z_{0} \equiv 0.21 \mathrm{pc}$ where $R_{0}=3.7 \times 10^{-3} \mathrm{pc}$. Saturation from an initial amplitude $A_{0} / R_{0}<<0.07$ can easily be achieved by $z=z_{0}+3.7 \mathrm{pc}(\lesssim 1$ mas $)$ where the saturation amplitude is $A / R_{j t}<0.025$.

\section{Summary and Conclusion}

The present work has shown that helical structure can be sensitive to jet and external medium conditions. This sensitivity manifests itself in predictable changes in wave speed, wavelength and growth rate along an expanding jet. The basic behavior of a non-disruptive "linear" helical twist is summarized below:

1. The wave speed varies, typically between limits set by the value of $\gamma a_{e x} / a_{j t}$ at low and $a_{j t}$ at high frequencies, relative to a resonant frequency, $\omega^{*} R_{j t} / a_{e x} \gtrsim 1.5$, at which the growth rate is largest.

2. The accompanying long and short wavelength regimes scale relative to the resonant wavelength $\lambda^{*} \propto\left\{\left[\gamma\left(M_{j t}^{2}-1\right)^{1 / 2}\right] /\left[\gamma\left(M_{j t}^{2}-1\right)^{1 / 2}+\left(M_{e x}^{2}-1\right)^{1 / 2}\right]\right\} M_{e x} R_{j t}$.

3. The spatial growth length scales $\propto \gamma M_{j t}$. A helical twist is damped along an expanding jet if $\gamma M_{j t}$ is too large.

4. Typically, a fixed frequency helical wave propagates from the low frequency to high frequency regimes by virtue of jet expansion.

5. Given equal excitation of multiple frequencies at a position $z_{0}$ the strongest helical pattern at $z$ will be at a frequency $\omega>\omega^{*}$ and wavelength $\lambda<\lambda^{*}$.

Modeling the detailed flow and pressure structure accompanying a helical twist shows the highest pressures to be near the surface of the jet. In general the jet fluid does not follow the helical path of the high pressure ridge. The correspondence between jet surface displacement, and pressure and velocity structure is summarized below:

1. A given pressure fluctuation induces a larger velocity fluctuation for higher jet sound speed.

2. Surface displacements accompanying similar levels of pressure fluctuation are larger for lower jet sound speeds but remain relatively small on relativistic flows.

3. Maintaining a saturation amplitude implies a decrease in the surface displacement as $\omega>\omega^{*}$.

4. At saturation amplitudes the jet center is at higher speed and lower pressure than the jet surface.

5. Axial velocity fluctuations are largest near the jet surface. Transverse velocity fluctuations are largest in the jet interior.

6. The maximum helical flow pitch angle is less than the relativistic Mach angle and always less than the helical pressure ridge pitch angle.

The present work shows how projection and light travel time effects profoundly influence the appearance of helical twisting for jets at small viewing angles. This extreme sensitivity should allow the determination of jet and external medium properties, albeit it is essential to have proper motion information in order to constrain the observed jet's speed and orientation. The brightest radio emission is expected to be generated in the region of highest pressure so observations will trace 
the path of the high pressure ridge. On an expanding relativistic jet the theoretically predicted patterns are like those that would be expected if the jet intensity were enhanced along a helical path on a conical surface. The brightest regions are where the helical path approaches closer to the viewing angle. The brightness is enhanced by foreshortening and by Doppler boosting, albeit by less than would be assumed from the pitch angle of the high pressure ridge. The observed appearance of a helical twist is summarized below:

1. Short wavelength twists in the high frequency regime travel at nearly the jet speed. On superluminal jets their apparent wavelength will be longer than the intrinsic wavelength.

2. For a fixed frequency helical wave the apparent wavelength change is amplified by ( 1 $\left.\beta_{w} \cos \theta_{0}\right)^{-1}$ relative to the intrinsic wavelength change $\propto \beta_{w}$. As a result $\lambda^{o b s}$ can appear $\propto R_{j t}$ as a jet expands if $a_{e x} \ll a_{j t}$.

3. Multiple frequencies can produce $\lambda^{o b s} \propto R_{j t}$ on average and can produce very different apparent motions at comparable locations.

The motion of components in the 3C 120 jet inside 10 mas may serve as an example of the predicted helical wave behavior on an expanding relativistic jet. At an assumed viewing angle of $9.6^{\circ}$ the observed component motions would indicate an isothermal jet expansion with jet sound speed $\approx 0.3 \mathrm{c}$ and sound speed in the medium immediately outside the jet $\gtrsim 0.1 \mathrm{c}$. Additional modeling work is planned to constrain the viewing angle and evaluate the robustness of these estimates.

It is anticipated that detailed modeling of observed helical structure on jets with observed proper motions will provide strong constraints on jet and external sound speeds, and on the perturbation frequencies that arise from or near to the central engine. Note that considerably different wave speed and wavelength behavior is associated with, for example, the present assumed isothermal as opposed to an adiabatic expansion. Thus, modeling results have direct implications for particle energization/heating rates and indirectly for particle acceleration rates. The finding of say high jet sound speed implies the lack of a "cold" baryonic jet component and may provide constraints on the composition of jet material, i.e., electron-proton dominated or electron-positron dominated as some polarization observations can be taken to imply (Wardle et al. 1998; Celotti et al. 1998).

The author would like to thank Jean Eilek, Andrei Lobanov and Craig Walker for motivating this study and providing illuminating discussions. The author would also like to thank JoseLuis Gómez for helpful comments and for providing an image of the inner 3C 120 jet. P. Hardee acknowledges partial support from the National Science Foundation through grant AST-9802955 to the University of Alabama.

\section{REFERENCES}

Agudo, I., Gómez, J.-L., Martí, J.-M., Ibáñez, J.-M., Marscher, A.P., Alberdi, A., Aloy, M.-A., \& Hardee, P.E. 2001, ApJ, 549, L183

Aloy, M.-A., Ibáñez, J.-M., Martí, J.-M., Gómez, J.-L., \& Müller, E. 1999, ApJ, 523, L125

Aloy, M.-A., Ibáñez, J.-M., Martí, J.-M., \& Müller, E. 1999a, ApJS, 122, 151

Aloy, M.-A., Gómez, J.-L., Ibáñez, J.-M., Martí, J.-M., \& Müller, E. 2000, ApJ, 528, L85

Aloy, M., Martí, J., Gómez, J., Agudo, I., Müller, E., \& Ibáñez, J. 2003, ApJ, 585, L109

Baldwin, J. A., Carswell, R. F., Wampler, E. J., Smith, H. E., Burbidge, E. M., \& Boksenberg, A. 1980, ApJ, 236, 388 
Biretta, J.A., Sparks, W.B., and Macchetto, F. 1999, ApJ, 520, 621

Bridle, A.H., \& Perley, R.A. 1984, ARA\&A, 22, 319

Clarke, D.A., Norman, M.L., \& Burns, J.O. 1989, ApJ, 342, 700

Duncan, G.C., \& Hughes, P.A. 1994, ApJ, 436, L119

Falle, S.A.E.G., \& Komissarov , S.S. 1996, MNRAS, 278, 586

Gómez, J. L., Martí, J.-M., Marscher, A.P., Ibáñez, J.-M., \& Alberdi, A. 1997, ApJ, 482, L33

Gómez, J.-L., Marscher, A.P., Alberdi, A., Martí, J.-M., \& Ibáñez, J.-M. 1998, ApJ, 499, 221

Gómez, J.-L., Marscher, A.P., \& Alberdi, A. 1999, ApJ, 521, L29

Gómez, J-L., Marscher, A.P., Alberdi, A., Jorstad, S.G., \& Garcia-Miró, C. 2000, Science, 289, 2317

Gómez, J.-L., Marscher, A. P., Alberdi, A., Jorstad, S. G., \& Agudo, I. 2001, ApJ, 561, 161

Hardee, P.E. 1984, ApJ, 287, 523

Hardee, P. E. 1987, ApJ, 318, 78

Hardee, P.E., Clarke, D.A., \& Rosen, A. 1997, ApJ, 485, 533

Hardee, P.E. 2000, ApJ, 533, 176

Hardee, P.E., Hughes, P.A., Rosen, A., \& Gomez, E. 2001, ApJ, 555, 744

Hardee, P.E., \& Hughes, P.A. 2003, ApJ, 583, 116

Hardee, P.E., \& Rosen, A. 1999, ApJ, 524, 650

Hardee, P.E., \& Rosen, A. 2002, ApJ, 576, 204

Hardee, P.E., Rosen, A., Hughes, P.A., \& Duncan, G.C. 1998, ApJ, 500, 599

Heinz, S., \& Begelman, M.C. 2000, ApJ, 535, 104

Homan, D.C., Ojha, R., Wardle, J.F.C., Roberts, D.H., Aller, M.F., Aller, H.D., \& Hughes, P.A. 2002, ApJ, 568, 99

Hughes, P.A., Miller, M.A., \& Duncan, G.C. 2002, ApJ, 572, 713

Jones, T.W., Ryu, D., \& Engel, A. 1999, ApJ, 512, 105

Koide, S., Nishikawa, K.-I., \& Mutel, R.L. 1996, ApJ, 463, L71

Lobanov, A., Hardee, P., \& Eilek, J. 2003, The Physics of Relativistic Jets in the CHANDRA and XMM Era, New Science Reviews, in press

Lobanov, A.P., \& Zensus, J.A. 2001, Science, 294, 128

Martí, Müller, \& Ibáñez 1994, A\&A, 281, L9

Owen, F.N., Hardee, P.E., \& Cornwell, T.J. 1989, ApJ, 340, 698

Reid, M.J., Biretta, J.A., Junor, W., Muxlow, T.W.B., \& Spencer, R.E. 1989, ApJ, 336, 112

Rosen, A., Hardee, P.E., Clarke, D.A., \& Johnson, A. 1999, ApJ, 510, 136

Rosen, A. \& Hardee, P.E. 2000, ApJ, 542, 750

Steffan, W., Zensus, J. A., Krichbaum, T. P., Witzel, A., \& Qian, S. J. 1995, A\&A, 302, 335

Synge, J.L. 1957, The Relativistic Gas, (Amsterdam:North-Holland)

Unwin, S.C., \& Wehrle, A.E. 1992, ApJ, 398, 74

Walker, R. C., Seielstad, G. A., Simon, R. S., Unwin, S. C., Cohen, M. H., Pearson, T. J., \& Linfield, R.P. 1982, ApJ, 257, 56

Walker, R. C., Benson, J. B., \& Unwin, S. C. 1987, ApJ, 316, 546 
Walker, R. C. 1997, ApJ, 488, 675

Walker, R. C., Benson, J. B., Unwin, S. C., Lystrup, M.B., Hunter, T.R., Pilbratt, G., \& Hardee, P.E. 2001, ApJ, 556, 756

Xu, C., Baum, S.A., O’Dea, C.P., Wrobel, J.M., \& Condon, J.J. 2000, AJ, 120, 2950

Xu, J., Hardee, P.E., \& Stone, J.M. 2000, ApJ, 543, 161

Zensus, J.A., Cohen, M.H., \& Unwin, S.C. 1995, ApJ, 443, 35 